"flat conuradiciun" to say that " unlike the cranium, however, the eyeball is not a rigid case," and that the stretching is " quite an appreciable amount," even when the intraocular pressure is raised from $19 \mathrm{~mm}$. to $70 \mathrm{~mm}$. Hg. The last statement is based upon reliable experimental observations by Koster. Dr. Henderson brings forward no evidence whatever to controvert these statements, and we must therefore conclude that, though within the range of physiological pressures, the walls of the globe may be regarded as practically a rigid case, beyond that range deformation occurs which must be taken into account if accurate deductions are to be made.

So far then as variations within the limited range of physiological pressures are concerned Dr. Henderson's working model of the circulatory mechanism of the intraocular pressure has exactly the same value that a good diagram has and no more. Those who have had experience in biological experiments will appreciate the dangers of mechanical working models. They have their use in illustrating proved facts; it is unwise to misuse them to prove disputed points. Within the narrow range of pressures in which the eyeball may be regarded as rigid the general application of Dr. Hill's researches may be freely admitted. Even they, however, should not be pressed too far. To take a simple example, his experiments tend to prove that vaso-motor changes cannot occur within the cranium. Delicate as were the experiments they appear to me to be far too coarse to prove such a point. As the late Professor Michael Foster said of experiments on cerebral localisation, "they are like playing the piano with a broomstick." Now there is plenty of evidence, clinical and experimental, to prove that vaso-motor changes occur in the retinal vessels within the normal range of intraocular pressures, yet Dr. Hill's experimental observations are equally potent in their allotted sphere. Under the conditions named the general features of the intraocular pressure are doubtless the same as those of the intracranial pressure, but only direct experiments on the eye can demonstrate what modifications in detail may be present. Beyond the given range one fact alone suffices to show that the conditions are quite dissimilar. When the animal is killed the intracranial pressure promptly sinks to zero; not so, however, the intra-ocular pressure, which, under these circumstances or when the eye is excised, only reaches zero after a prolonged period-in fact, after fluid has filtered out.

With regard to the production of aqueous Dr. Henderson adopts the secretory view in common with many who hold what he calls the "volumetric" theory. They, however, do not go so far as to say that " mechanical filtration is impossible." It is difficult to understand what he means when he says that "there can be no difference in hydrostatic level on either side of the filtering membrane." The filtering membrane is the capillary wall, and whatever may be the reason why the fluid passes into the posterior chamber and not into the suprachoroidal space, it is certain that the intracapillary pressure is greater than the extracapillary-i.e., the intraocular-pressure, and therefore the filtration factor must come into play. Resorption of aqueous is regarded as a pure diffusion into the veins, particularly the canal of Schlemm and the veins of the iris. Dr. Henderson believes that many of the ciliary vessels which have been described as arteries are really veins.

On the assumption that even under pathological conditions of increased tension the walls of the eye are still a rigid case, the explanation of glaucoma is held to be due, not to a "retention of intraocular volume," but to a "retention of intraocular pressure." The language is cryptic, but taken with the context the meaning is clear. On blocking the venous exits-canal of Schlemm by sclerosis of the ligamentum pectinatum and anterior ciliary veins by peripheral anterior synechia-the elastic blood-vessels are transformed into a rigid system of tubes. In such a system the ontflow pressure is always higher than in a similar system of elastic tubes; hence in glaucoma the lowest circulatory pressure is that in a rigid system, and therefore the intraocular pressure is maintained at a correspondingly high level. The observations on sclerosis of the ligamentum pectinatum agree with those of other pathologists. Resorption of aqueous is reduced to the area of the iris veins. The aqueous is therefore not entirely cut off from the veins; the aqueous volume is displaced by increase of total vascular volume, so that the anterior chamber becomes shallow. The explanations of the dilatation of the pupil, haziness of the cornea, occlusion of the angle of the anterior chamber, and so on, are very ingenious, but are open to criticism in many respects.

The efficacy of iridectomy in glaucoma is attributed to the free opening up of access to the iris veins. This is facilitated by the fact that wounds of the iris do not undergo cicatrisation like other wounds, and consequently the aqueous is brought into contact with the vessels of the iris throughout the length of both edges of the coloboma.

That the hydrostatic principles underlying Dr. Henderson's theory have to be taken into account needs no proof, and he has done great service in forcing them vigorously upon the attention of ophthalmologists. That there are conditions, normal and pathological, in which they play a preponderant rôle may equally be admitted. That they alone can be held to account for the pathogenesis of glaucoma is a view unlikely to commend itself to most pathologists, who will still hold that the major variations in the intraocular pressure are brought about by actual variations in the fluid contents of the globe. Further research will probably show that the two theories are not so inconsistent as Dr. Henderson would have us believe.

There yet remains an operation for chronic glaucoma which has not been considered-cyclodialysis. By this means Dr. Heine attempted to provide an exit of the intraocular fluid by way of the suprachoroidal space. It appears to be satisfactory so long as the artificial detachment of the ciliary body from the sclerotic persists, but fibrous reunion occurs and the intraocular tension again rises. According to Dr. Henderson there is normally free passage of aqueous from the anterior chamber into the suprachoroidal space; his evidence, however, is not convincing. $\mathrm{Dr}$. A. Freeland Fergus combines the operation of trephining with cyclodialysis.

In conclusion, if the criticisms put forward here are just, the formation of a filtering cicatrix holds out the best hope of alleviating chronic glaucoma.

Queen Anne-street, W.

\section{LYMPHATIC VARICOCELE.}

\section{BY FRANK COLE MADDEN, M.D. MELB., F.R.C.S. ENG.}

PROFESSOR OF SURGERY, EGYPTIAN GOVERNMENT SCHOOI OF MEDICINE; SENIOR SURGEON, KASR-EL-AINY HOSPITAL,

$$
\text { CAIRO. }
$$

WE have recently operated on three cases presenting the usual clinical signs and symptoms of varicocele, but have found that the swelling was due to the presence of tortuous coils cf very thin-walled, dilated lymphatics in the substance of the spermatic cord. This condition is, in all probability, a manifestation of filariasis, though we have not succeeded in finding filarial embryos in the blood-a fact that proves nothing of itself, but it would have been a source of satisfaction to have had confirmation of our thesis.

Our attention was first drawn to the condition I wish to describe whilst operating on a case which had been operated on a few months before for varicocele. A very temporary relief had followed the first operation, but the swelling had returned in a more severe degree shortly afterwards. At the second operation we found that the varicocele had been completely cured-if, indeed, it had ever existed; but we were surprised to find masses of thin, translucent, dilated lymphatics extending along the whole length of the cord. The affected lymphatics were excised, and so far there has been no recurrence, though, if the enlargement is due to a compensatory dilatation to provide a collateral circulation around a blocked lymphatic trunk in the pelvis or lower abdomen, the permanency of the cure is very uncertain.

The following case brings out the main points of the condition and its liability to recur, and may be taken as a type.

The patient, aged 20, was admitted to Kasr-el-Aing Hospital on Sept. 10th, 1911, complaining of pain in the right side of the scrotum. He had been suffering from this pain for the last five years, especially after prolonged standing or walking, and it was gradually increasing in severity. There was no history of fever or any other previous disease, either in the scrotum or elsewhere, and the urine was clear, with specific gravity 1022 , acid in reaction, and entirely 
free of albumin, sugar, or other abnormal constituent. No filarial embryos were found in his blood. He had been operated on in February, 1911, by my colleague, Dr. Aly Ibrahim, for hernia and varicocele, but the veins were not enlarged, and running with the hernial sac was a "cystic enlargement of the cord extending as far as the external ring. The thickening consisted of separate cystic enlargements, each of which was constricted at certain points, as though valves were present in the course of the dilated vessels." The true nature of the enlargement was not then recognised, but the cystic dilatations were excised between ligatures, and the radical operation for hernia was completed.

Very soon after this operation the swelling of the cord returned, and this was thought to be a thrombosis of the veins of the cord which would probably gradually subside. The swelling, however, remained stationary, and on readmission to hospital the enlargement very closely resembled varicocele, even giving a faint impulse on coughing, and diminishing in size on lying down and refilling on standing.

A second operation was performed. Under stovaine anæsthesia the old scar was resected, and the entire length of the cord was exposed from the external ring to the testis. The swelling was then seen to consist of a large number of coils of dilated lymphatics having a sacculated appearance, like sausage-skins of very small calibre, and distended with clear lymph. These coils completely surrounded the other constituents of the cord and extended into the abdomen. The veins were not affected. The mass of lymphatics was removed between ligatures above and below, and the operation was completed as though the mass had consisted of dilated veins. The scrotum was stitched up vertically in its upper part and transversely below to shorten the pendulous scrotum. After removal it was seen that the lymphatios formed long coiled tubes, held lightly together at some points by loose connective tissue. The subsequent history of the case was uneventful, and the enlargement has not yet returned.

This condition adds yet another to the already lengthy list of possibilities in the scrotum, and if it is a filarial manifestation, which it almost certainly is, it is further of interest on account of its restricted distribution. I do not remember to have read any report of filarial lymphatic dilatation strictly limited to the lymphatics of the spermatic cord, without any implication of the scrotum, either superficial or deep.

I am much indebted to Dr. Abd-el-Fataah, resident surgical officer, for the notes of this case.

Note.-In operating on hernia and varicocele one not infrequently finds thick, white, tenacious masses of coagulated lymph in and around the spermatic cord, very similar to the more organised lymphy tissue which has to be stripped off the cords in the operation for elephantiasis of the scrotum. This is probably due to exudation of lymph from increased lymph pressure in the lymphatics of the cord and surrounding connective tissue, and is not necessarily filarial at all. It is quite different from the condition above described and no appreciable dilatation of lymphatics is present.

Cairo.

\section{SOME OBSERVATIONS ON}

\section{THE PREVENTION OF TUBERCULOSIS.}

BY J. SIM WALLACE, M.D., D.Sc. GLasG., L.D.S., DHATAL SURGEON AND LECTURER ON DENTAL SURGERY AND PATHOLOGY TO THE LONDON HOSPITAX.

GENERALLY speaking, the healthy individual is an asset to the community, while the diseased are liable to be a burden. It would, therefore, seem to be to the advantage of the community to prevent disease. The treatment of disease is often quite a different matter. Once a disease, such as tuberculosis, effects an entry into the system, not only may the individual be completely incapacitated for work, but ouring the disease is problematical, even with the aid of lavish expenditure. The extreme tenacity with which disease germs keep hold of their host once they have gained an entrance into it was well brought out in a recent address by Sir Hector Cameron, ${ }^{1}$ but this fact is well known, and further reference to it is unnecessary here.

In days gone past the difficulty of expense in treatment

The Persistence of Disease Germs in the Human Body, Brit. Med Jour., 1911, vol, i., p. 973. was no doubt got over by the victim of such diseases entering an early grave; but it being impossible to countenance such a course of events now, we must consider whether it would not be profitable to direct more attention to prevention. It is particularly necessary to do so because the incubas of taxation for the treatment of tuberculosis and other diseases probably is a fruitful cause of tuberculosis and many other diseases.

There are two principal ways in which tuberculosis may be prevented : (1) The environment of the individual may be kept free, relatively at least, from germs which are liable to cause the disease ; (2) the individual may be kept sufficiently healthy to resist the effective entrance of disease germs. That the first method is important, especially in the more infectious diseases, is of course recognised. In the case of tuberculosis it is not so obvious, but that it is so is noticeable when the question is gone into completely. ${ }^{2}$ I do not intend, however, to deal with this part of the subject. It is to the possibility of more effectually keeping the body suffi. ciently fit to be able to resist the tubercle bacilli that I intend to direct attention, because the recommendations about to be made are as yet relatively unknown.

The two chief routes of entry of the tubercle bacilli into the system appear to be viâ the alimentary canal and the faucial and pharyngeal tonsils. With regard to entrance via the alimentary canal, we know that in a healthy state entrance in this way is hardly to be expected, and, as a matter of fact, though all people must swallow living tubercle bacilli many times during their life-perhaps, indeed, many times during each day-yet the tubercle bacilli do not by any means always gain effective entrance into the system.

In the past-attention being directed exclusively to the consideration of the nutritive values, the number of "calories," the rapidity of digestion, the assimilability of the food, \&c.-no real consideration seems to have been given to a most important, perhaps the most important, principle of dietetics-viz., the principle which claims that the meals should be so arranged that the mouth and alimentary canal shall be left in a hygienic state at the end of each meal.

With regard to the mouth, it may be said that but few conld indicate with the slightest degree of accuracy the natire of the foods which tend to leave the month in a hygienic state. Yet this is the beginning of the trouble; for if the mouth is, generally speaking, in an unhygienic state after meals -as it, indeed, very generally is - then what chance has the stomach and alimentary canal to be free from injury from the constant swallowing of septic matter, polluted food, or decomposing food remains. Furthermore, if the mouth is left in an unhygienic state, what chance have the teeth of remaining free from caries or the gums from being injured between the teeth by the more or less constant lodgment of fermenting food.

We now know that dental diseases are, in general, neither hereditary nor constitutional, but that they are the result of habitual dietetic error. The dietetic error which brings on dental troubles has little or nothing to do with the question as to whether the food is nutritious or not. The point of importance is whether the food leaves the mouth in a hygienic state at the end of the meal or otherwise. It is not my intention to consider which foods are cleansing to the mouth and teeth and which are not. Here it must simply suffice to say that those physicians who have really considered the subject now recognise that soft milk-soaked foods do not keep the mouth in a hygienic state, and moreover some note the probability that these foods are neither directly nor indirectly conducive to the hygiene of the alimentary canal. Thus, Dr. G. A. Sutherland, when referring to the preventive treatment of tubercular peritonitis, says that " the real risk lies in an unhealthy condition of the alimentary canal which may allow the penetration of the bacilli." He urges the wisdom of a type of diet requiring mastication such as would tend to keep the alimentary canal in a healthy state, and comments on the feeding of children with tubercular tendencies on "a sloppy diet-milk, bread and milk, and pudding." He says : "Experience has shown that this is the worst possible form of treatment." 3

Further somment on the system of feeding which keeps the mouth and alimentary canal in an unhygienic state is not

2 Newsholme: Prevention of Tuberculosis. 3 The Treatment of Diseases in Children, p. 110. 